\title{
A Novel Genetic Resource Allocation Algorithm for Symmetrical Services in OFDMA Systems
}

\author{
Swapna P. S., Sakuntala S. Pillai, Syama Sasikumar, Sreeni K. G.
}

\begin{abstract}
Performance enhancement of symmetrical services has been very essential today owing to the widespread acceptance and demand of these services in the present generation communication systems. An algorithm with reduced complexity for subcarrier allocation in OFDMA/SC-FDMA system for specific applications that demand similar bidirectional quality is proposed in this paper. The resource allocation problem devised is a multiobjective optimization problem with objectives to maximize bidirectional data rates and minimize the difference in bidirectional data rates, with fairness as a significant constraint. The original problem is mathematically intractable due to non-convexity and therefore linear programming techniques fail to find an optimal solution. The subcarrier allocation problem has been undertaken using an innovative multiobjective optimization technique that employs the concept of non-dominance in evolutionary algorithms. The results are extremely encouraging, while significantly reducing the complexity involved in the processing of algorithm.
\end{abstract}

Keywords: Evolutionary algorithm, Multiobjective Optimization, Non-dominance, OFDMA/SC-FDMA

\section{INTRODUCTION}

The telecommunication industry has changed dramatically over years as the number of customers demanding high data rate increase at an exponential rate. The increase in wireless utilization is tremendously coming up against the constraints of radio spectrum availability and telecommunication infrastructure. Another major issue in wireless communication is multipath fading, generating Inter symbol Interference (ISI), a major limitation for high data rate communication.

Due to the geographical spread of the OFDMA system users they observe independent fading parameters and thereby mitigating the effects of multipath fading and reducing the probability of a subcarrier to be in deep fade for all users. However the available subchannels are to be allocated adaptively to the users to meet their QoS. Subcarrier allocation schemes are basically classified into fixed and adaptive allocation techniques. Fixed allocation scheme works on the principle of allocating fixed number of

Revised Manuscript Received on December 03, 2019

* Correspondence Author

Swapna P. S.*, Dept. of ECE, Mar Baselios College of Engineering and Technology, Trivandrum, India. Email: swapna.ps@mbcet.ac.in

Sakuntala S. Pillai, Dept. of ECE, Mar Baselios College of Engineering and Technology, Trivandrum, India. Email: sakuntala.pillai@gmail.com

Syama Sasikumar, Dept. of ECE, Mar Baselios College of Engineering and Technology, Trivandrum, India. Email: syamanair02@gmail.com

Sreeni K. G., Dept. of ECE, College of Engineering, Trivandrum, India. Email: sreenikg79@gmail.com subcarriers to users without considering the current channel condition while adaptive allocation scheme allocates the subcarriers to the users adaptively, in an optimum manner to increase the performance of each user.

The Resource Allocation (RA) problem is usually approached in two ways: either for maximization of the data rate for a given transmit power termed as rate adaptive approach or for minimization of the transmit power for a desired data rate termed as margin adaptive approach Literature review shows that dynamic resource allocation enhance the system output in terms of throughput, spectrum usage, power consumption etc. to a large extent.

\section{A. Existing Works}

State of the art applications of smart phones like mobile gaming, video conferencing etc. have gained widespread popularity and demand symmetrical quality in both the uplink and downlink directions. Taking into consideration the above mentioned requirements, the RA problem formulated results in a multiobjective optimization problem. A number of techniques have been investigated in literature to address the issue of RA for symmetrical services in OFDMA system [1]-[10]. The problem has been addressed using optimization theory, graph theory and game theory. Since the RA problem for improving the performance of the system with symmetrical services, is a constrained multiobjective optimization problem, the use of linear programming techniques results in high order of complexity [4]. Reference [9] makes use of game theory for resource allocation and interference mitigation. In all the above mentioned techniques, the order of complexity of the solution was not considered and all ended up in highly complex solutions which cannot be implemented for real time services. Therefore the need for solutions with low complexity becomes necessary for real time applications.

\section{B. Contributions}

Maximizing each users bidirectional data rate and minimizing difference in the bidirectional data rates for each individual user are the key objectives of the devised optimization problem. Since the complexity of the solution is considered of prime significance we take the application of an equal power allocation technique, since it provides results in par with water filling algorithm at high SNR[10].

For subcarrier allocation, Genetic algorithm (GA) is adopted since it provides better results compared with linear programming techniques, 


\section{A Novel Genetic Resource Allocation Algorithm for Symmetrical Services in OFDMA Systems}

in addition to reducing the complexity of implementation. Moreover GAs are seldom used to solve such optimization problems where symmetrical services are required. Non-dominance Sorting Genetic Algorithm II (NSGA-II) is the most popular multiobjective optimization algorithm in the category of evolutionary algorithms and is being employed here for subcarrier allocation.

To summarise:

- We first consider maximizing the data rates and minimizing difference in uplink/ downlink throughput for individual user in the system, under the assumption that all system users require symmetrical quality.

- We have imposed fairness as a significant constraint during problem formulation.

- NSGA II has been adopted for finding a less complex solution to the multiobjective optimization problem.

- We have conducted extensive simulations, which revealed less complex optimal solutions are possible in par with other techniques.

\section{FORMULATION OF RESOURCE ALLOCATION PROBLEM}

The algorithm is applied at the transmitter of both uplink and downlink directions of SC-FDMA and OFDMA systems respectively, to allocate the subcarriers to the users in a manner that the user attains almost similar bidirectional data rates. Equal power allocation is considered in this solution. An update is provided to base station and user equipment, whenever there is a change in the sub-channel information.

A single cell OFDMA system for downlink and SC-FDMA in uplink is considered. The cell spectrum is split across different sub bands, each supported by a subcarrier. The total bandwidth of $\mathrm{B} \mathrm{Hz}$ is divided into a set of $\mathrm{M}$ subcarriers and shared by a set of $\mathrm{K}$ users. The maximum power constraint is considered as $\mathrm{P}_{\mathrm{T}}$. No two user can share the same bandwidth due to interference issues. Through feedback channels the information regarding the channel states (CSI) is provided to the base station and user equipment. To support this assumption, a flat faded Rayleigh channel is considered.

The transmission process comprises of the following phases: 1) uplink phase 2) downlink phase.

1) Uplink Phase

In this phase, the data from the user is sent through an SC-FDMA system to the base station. There are $\mathrm{M}$ number of subcarriers which are to be allocated among $\mathrm{K}$ users. For $i^{\text {th }}$ user and $\mathrm{j}^{\text {th }}$ subcarrier, $\mathrm{h}_{\mathrm{ij} 2}$ is the channel gain. Signal to noise ratio is therefore $\frac{h_{i j 2}^{2}}{\operatorname{No} B / N}$ and is denoted by $\gamma_{i j 2}$, where $\mathrm{B}$, No, and $\mathrm{N}$ are the bandwidth, noise power spectral density and noise power, respectively. $\omega_{\text {iij } 2}$ is the subcarrier allocation metric and it takes the value 1 if $\mathrm{j}^{\text {th }}$ subcarrier is allocated to $i^{\text {th }}$ user or else it is set to 0 . Data rate of $i^{\text {th }}$ user in uplink is denoted by $\mathrm{R}_{\mathrm{i} 2} \cdot \mathrm{P}_{\mathrm{T}}$ is the total power allocated for uplink direction.

2) Downlink Phase

In this phase, the data from the base station is passed through an OFDMA system to the user. There are M number of subcarriers which are to be allocated among $\mathrm{K}$ users. For $\mathrm{i}^{\text {th }}$ user and $\mathrm{j}^{\text {th }}$ subcarrier, $\mathrm{h}_{\mathrm{ij} \mathbb{1}}$ is the channel gain. $\frac{\mathrm{h}_{\mathrm{i} \mathrm{I}}^{\mathrm{T}} \mathrm{I}}{\mathrm{No} \cdot \mathrm{B} / \mathrm{N}}$ is the signal to noise ratio and is denoted by $\gamma_{\mathrm{i} j \mathbb{1}}$, where $\mathrm{B}$, No, and $\mathrm{N}$ are bandwidth, noise power spectral density and noise power, respectively. $\omega_{\mathrm{i} i 1}$ is the subcarrier allocation metric and it takes the value 1 if $\mathrm{j}^{\text {th }}$ subcarrier is allocated to the $\mathrm{i}^{\text {th }}$ user, else it is set to $0 . \mathrm{P}_{\mathrm{BS}}$ is the total power allocated for downlink direction.

The objectives of the problem are

Maximize $\mathrm{R}_{\mathrm{i} 1}$

Maximize $\mathrm{R}_{\mathrm{i} 2}$

Minimize the difference in the magnitudes of the two rates of user i i.e., $\left\|R_{i 1}-R_{i 2}\right\|$

The expression for $R_{\tilde{1} \mathbb{1}}$ is given as

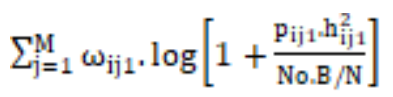

Similarly expression for $\mathbb{R}_{\mathrm{i} 2}$ is

$$
\sum_{i=1}^{M} \omega_{i j 2} \cdot \log \left[1+\frac{P_{i j 2} \cdot h_{i j 2}^{2}}{N o \cdot B / N}\right]
$$

The third objective is to minimize the difference in the rates of the $i^{\text {th }}$ user i.e., Minimize $\left\|R_{\mathrm{i} 1}-R_{\mathrm{i} 2}\right\|$, with the following constraints,

$\mathrm{C} 1: \sum_{\mathrm{j}=1}^{\mathrm{M}} \omega_{\mathrm{ij} 1} \leq 1$, for the downlink direction

C2: $\sum_{\mathrm{j}=1}^{\mathrm{M}} \omega_{\mathrm{ij} 2} \leq 1$, for uplink direction

$$
\mathrm{C} 3: \omega_{\mathrm{ij} 1}, \omega_{\mathrm{ij} 2} \in[0,1]
$$

$\mathrm{C} 4: \Sigma_{\mathrm{j}=1}^{\mathrm{K}} \mathrm{P}_{\mathrm{ij} 1}=\mathrm{P}_{\mathrm{T}} / \mathrm{k}_{v}$ for uplink

$$
\mathrm{C} 5: \sum_{\mathrm{j}=1}^{\mathrm{K}} \mathrm{P}_{\mathrm{ij} 2}=\mathrm{P}_{\mathrm{BS}} / \mathrm{k}_{\mathrm{s}} \text { for downlink }
$$

$$
\text { C6: } \mathrm{R}_{\mathrm{i} 1} \geq \mathrm{Rmin}
$$

C7: $\mathrm{R}_{\mathrm{i} 2} \geq \mathrm{Rmin}$

The constraints $\mathrm{C} 1, \mathrm{C} 2$ and $\mathrm{C} 3$ ensure single user allocation of a particular subcarrier in the respective direction. The constraints $\mathrm{C} 4$ and $\mathrm{C} 5$ show that the total power is equally distributed among the users. The constraints C6 and C7 are imposed for ensuring fairness in the system. Being non-convex in nature, this belongs to the set of mixed integer programming problems which suffer from high computational complexity. Therefore employing linear programming techniques lead to a solution which is highly complex. This has motivated us to look for other techniques and thereby arriving at solutions using genetic algorithm. 


\section{PROPOSED SOLUTION}

In this work, NSGA II is utilized to allocate the subcarriers in an OFDMA/SC-FDMA system for symmetrical services with equal power allocation. Since the RA problem formulated is multiobjective in nature, the fitness functions are multiple, namely the data rates in uplink and downlink and the difference between the two for each user. The steps followed for allocation is as follows:

Step 1: Number of generations (G), Size of population, Channel gain ' $h$ ' for each user in each subcarrier, Total Power (PT), Bandwidth (B), number of subchannels (M), number of users $(\mathrm{K})$, and ${ }^{R m i n}$ are provided as inputs.

Step 2: Initial population of chromosomes are created and their data rates are calculated using (1) and (2) and the difference in data rates. The chromosomes are represented as a string of non-binary numbers. For example, in a string ' 1122 ' since the first position is a 1 , it means that the first subcarrier is allocated to user 1. Similarly, the value in the next position is also 1 , indicating that the second subcarrier is allocated to user 1 . The third and fourth position has a value ' 2 ' which means that the third and fourth subcarrier is allocated to user 2 . This kind of representation also aids in the reduction of the complexity of the solution to a large extent.

Step 3:The fitness function for each chromosome. ie., value of Ri1 and Ri2 and the difference in data rates is then computed.

Step 4: The chromosomes are sorted in a manner that all solutions that are not dominated by any other into front 1 , and dominated by any one into front 2 and so on.

Step 5: Probability levels are generated for each chromosome depending on fronts allotted, such that the chromosome in front 1 has the best chance for crossover selection.

Step 6: Two chromosomes are selected based on fitness value from the entire population. Two point crossover is opted in this solution. If the chromosomes selected are 1112 and 2221, then in two point crossover, two points are randomly selected. For example, if 1 and 3 are selected, then after the first crossover the resultant is 2112 and 1221 . When the crossover is carried out in position 3, it becomes 2111 and 1222. So obtained is the child chromosome after crossover.

Step 7: The two new child chromosomes termed as offspring are used to replace the worst two chromosomes.

Step 8: From the entire population a chromosome is selected randomly for mutation.

Step 9: The channels allocated to users in the selected chromosome is flipped.

Step 10: To ensure fairness of the system, if a particular user has more channels and another user has not attained the minimum number of channels, then required number of channels are reallocated so that all users meets the minimum requirement.

Step 11: The data rates for each chromosome is calculated using Eq. (1 \& 2).

Step 12: Iterate till the number of generations is reached.

\section{SIMULATION RESULTS}

A Rayleigh fading channel is assumed between the source and destination. Maximum delay spread of $5 \mu \mathrm{s}$ has been assumed and the CSI is updated every $0.5 \mathrm{~ms}$. The total bandwidth is assumed to be $0.5 \mathrm{MHz}$ for a user and total number of subcarriers as 4 in figure 2 and 3 . These constants are used for the calculation of the data rates in uplink and downlink direction.

The evaluation of the proposed algorithm is described in three sections. The first section depicts the variation of the objectives with the number of generations. The second section illustrates the variation of the data rate with the SNR for a 2 user system. The third section shows the deviation of the objectives with the generations for a 12 user system with 64 subcarriers. The simulation results obtained after applying NSGA II for subcarrier allocation is shown in the following figures.

Fig. 1(a) clearly depicts that the data rates for two users in the uplink / downlink direction computed from initial population is much lower than the other generations. This is due to the fact that the initial population was randomly chosen and the data rates achieved may or may not be good ones with this population. Fig.1 (b) shows that the data rates increase with generations and data rates exhibit almost similar values as the number of generation's increases. This can be justified by the fact that after computing the fitness function in each generation, they are sorted according to non-dominance criteria. As the generations proceeds, all possible combination of chromosomes are tried out and the best values are attained. Since one of the objectives of the optimization problem was to minimize the difference in data rates, the difference is within limits irrespective of the number of generations. For higher value of generations the difference in data rates for both uplink and downlink direction are very small and consistent. This is illustrated in Fig. 1(c). The results obtained are comparable to those using LPP techniques, for equal power allocation. Moreover the results obtained are computationally less expensive compared to LPP techniques. From the figure the difference in the data rates is seen to be reduced compared to that of the initial population and an increase in data rates as expected is seen.

Fig. 2 shows the change of the average data rates with SNR. As the signal power increases data rates also increase accordingly.

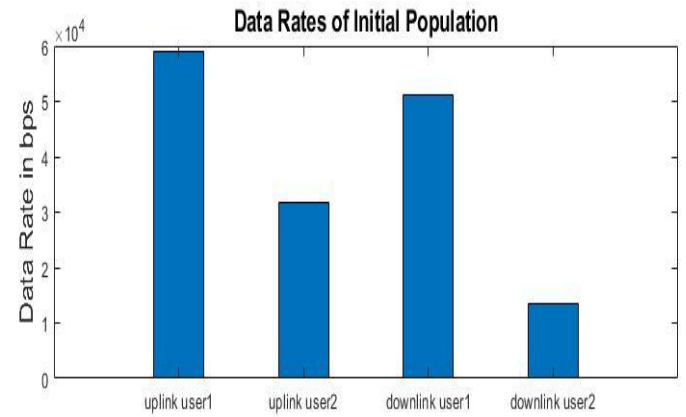

(a) 


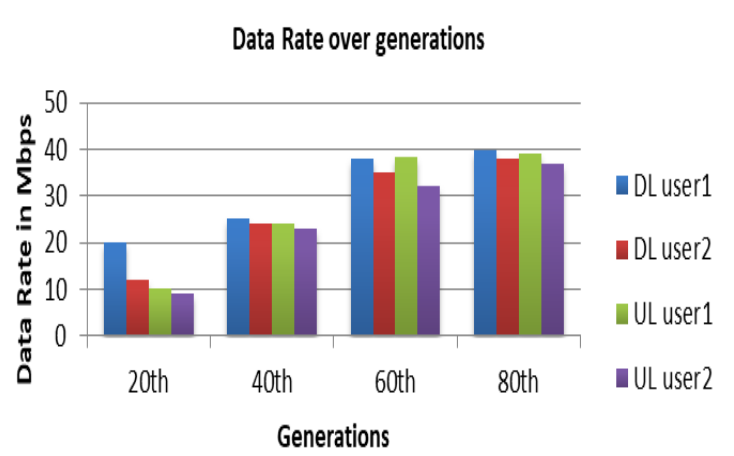

(b)

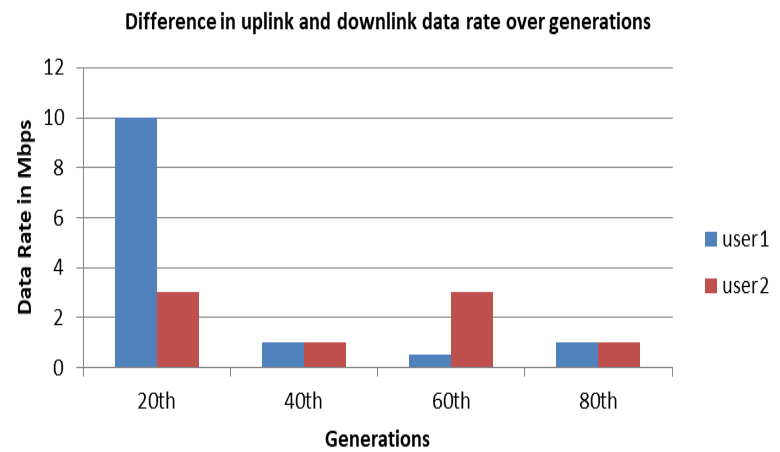

(c)

Fig.1: Simulation results for a 2 user system for various generations

Fig. 3(a) shows the computed fitness value for the initial population of 12 users and 64 subcarriers. Fig. 3(b) depicts the system performance for 12 users and 64 subcarriers in the 80th generation.

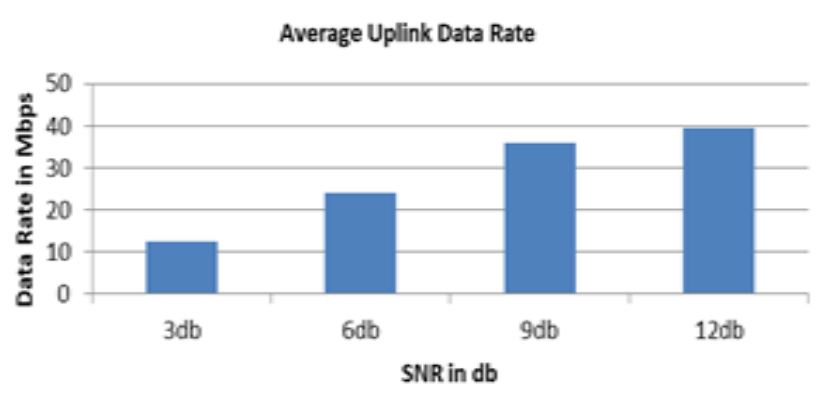

(a)

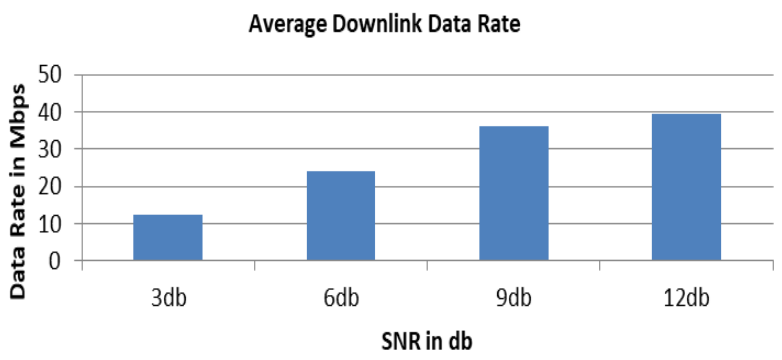

(b)

Fig.2: Simulation results for a 2 user system for various SNR

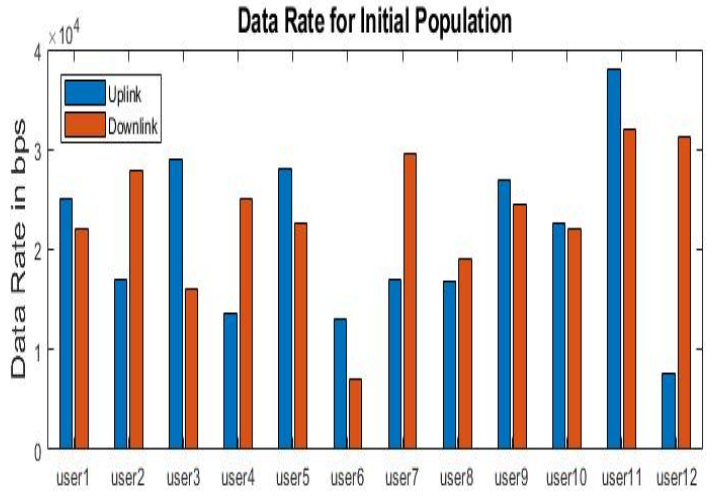

(a)

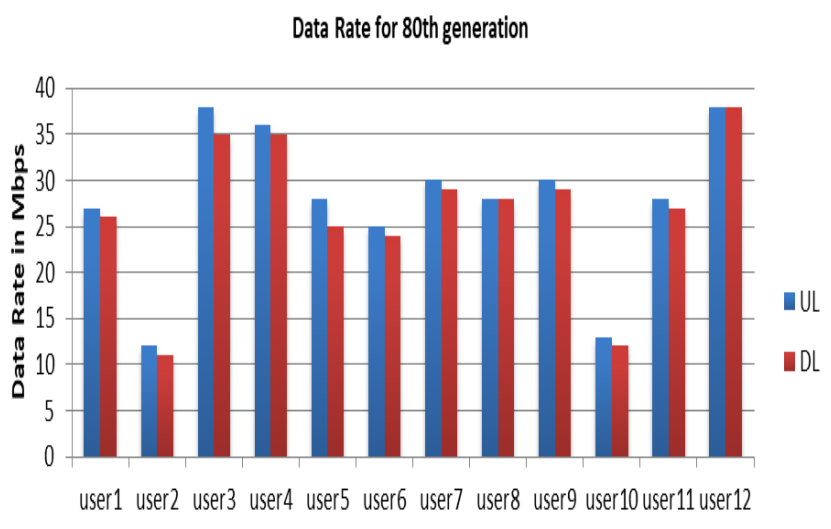

(b)

Fig.3: Simulation results for a 12 user system

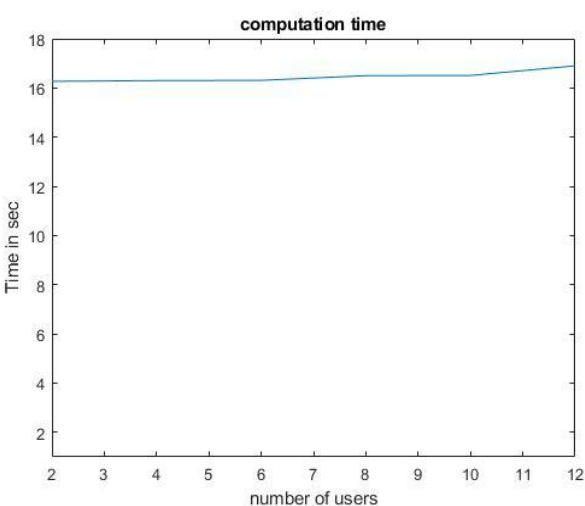

Fig.4: Computation Time for varying number of users in the 80th generation

Fig. 4 shows the time taken for the algorithm to process the 80th generation for varying user numbers and 64 subcarriers. Since the chromosomes were represented as a string of non-binary numbers the total number of multiplications depends only on the number of subcarriers which is indeed the length of the string and does not depend on the number of users. This is unlike the linear programming techniques where the complexity of the algorithm is of the order of product of user numbers and subcarrier numbers

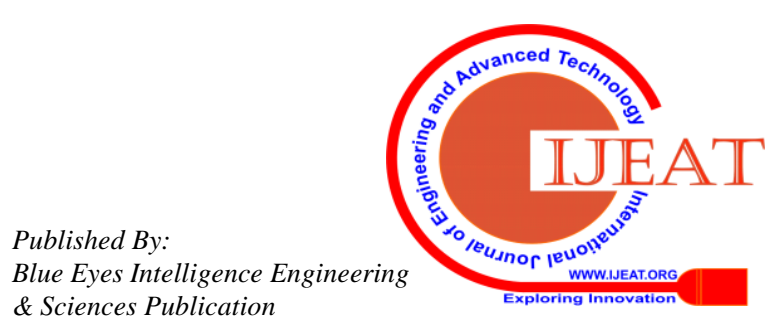




\section{CONCLUSION}

In this paper, we proposed NSGA II as the solution for subcarrier allocation for symmetrical services in OFDMA/SC-FDMA system with equal power allocation. Considering the real time applications of wireless system, the complexity of the techniques is to be taken into prior importance. Thereby we propose a resource allocation technique, where NSGA II has been adopted for subcarrier allocation and equal power allocation. To this end, we have shown that the computational complexity depends primarily on the number of subcarriers and not on the product of user numbers and subcarrier numbers as in the techniques discussed in literature. The simulation results shows acceptable range of downlink and uplink data rates and the difference between the bidirectional data rates are within the threshold level.

\section{REFERENCES}

1. Ahmad M. El-Hajj, Zaher Dawy, "On Probabilistic Queue Length based Joint Uplink/Downlink resource allocation in OFDMA networks", 19th International Conference on Telecommunications 2012.

2. Ahmad M El-Hajj, Zaher Dawy and Walid Saad, "A stable matching game for joint Uplink/Downlink resource allocation in OFDMA wireless networks", IEEE ICC 2012-Wireless network Symposium.

3. Ahmad M El-Hajji, Zaher Dawy, "On optimized joint uplink/downlink resource allocation in OFDMA networks", 2011 IEEE Symposium on Computers and Communications.

4. Ahmad M El-Hajji, Mariette Awad, Zaher Dawy, "SIRA:A socially inspired game theoretic uplink/downlink resource aware allocation in OFDMA systems", 2011 IEEE International Conference on Systems, Man and Cybernetics.

5. Lukai Xu, Guanding Yu and Yuhuan Jiang,"Energy Efficient Resource Allocation in Single Cell OFDMA Systems: Multi-Objective Approach", IEEE Transactions on Wireless Communications,2015.

6. Lukai $\mathrm{Xu}$, Guanding $\mathrm{Yu}$, Yuhuan Jiang and Qimei Chen, "Multi-Objective Bandwidth and Power Allocation for Energy-Efficient Uplink Communications", 2015 IEEE 26th Annual International Symposium on Personal ,Indoor and Mobile Radio Communications.

7. Zhengyu Song, Qiang Ni, Keivan Navaie, Shujuan Hou and Siliang Wu, "Energy and Spectral Efficiency Tradeoff with $\alpha$-Fairness in Downlink OFDMA Systems",IEEE Communications Letters, 2015.

8. U. Akgul, B. Canberk, 'Self-Organized Things (SoT): An Energy Efficient Next Generation Network Management', Computer Communications (Elsevier), vol. 74, pp. 52-62, January 2016.

9. Guopeng Zhang, Kun Yang, and Hsiao-Hwa Chen, "Resource Allocation for Wireless Cooperative Networks - A Unified Cooperative Bargaining Game Theoretic Framework", IEEE Wireless Communications, Volume: 19, Issue: 2, Page(s): 38-43, April 2012. (SCI).

10. Elias Yaacoub and Zaher Dawy. "A survey on uplink resource allocation in OFDMA wireless networks". IEEE Communications Surveys \& Tutorials,14(2):322-337, 2012.

\section{AUTHORS PROFILE}

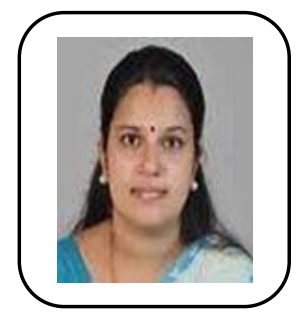

Swapna, in 2007, obtained Master of Technology (M.Tech) in Microwave and Television Engineering from College of Engineering, Trivandrum, Kerala. In 2004, she graduated in Electronics and Communication Engineering from Rajeev Gandhi Institute of Technology Government Engineering College, Kottayam, Kerala. Whilst pursuing Ph.D from Kerala University, she is also working currently as Assistant Professor at Mar Baselios College of Engineering and Technology, Trivandrum, Kerala in Electronics and Communication Engineering department. She has 14 publications in her chosen research area.
Her areas of interest include resource allocation for OFDMA systems, Resource management for $5 \mathrm{G}$ networks etc...She has a total teaching experience of 12 years.

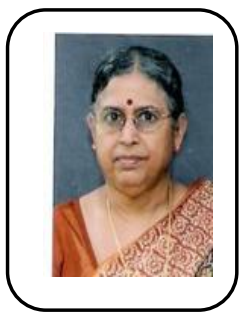

Dr. Sakuntala, in 1989, obtained Ph. D degree from University of Kerala. Having served as Head of Department of Electronics and Communication, College of Engineering, Trivandrum from 1996-1998, she worked as Director, LBS centre for Science and Technology, Trivandrum. From 2003, she worked in Mar Baselios College of Engineering \& Technology, Trivandrum as Head the Department of Electronics \& Communication and later served as Dean (R\&D) from 2009-2016.

Her research interests include, OFDMA, OFDM, Error Correction Coding, MIMO wireless systems, etc are few of her research interest areas. She has more than 60 publications in this area. She is a Fellow of Institution of Engineers (India), Fellow of IETE and Senior Member of IEEE.

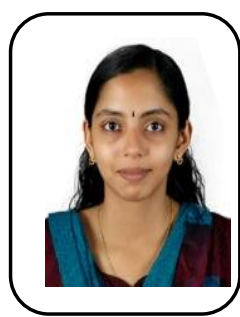

Syama, in 2015, received M.Tech in Telecommunication Engineering from Mar Baselios College of Engineering and Technology, Trivandrum, Kerala. In 2012, she graduated in Electronics and Communication Engineering from PRS College of Engineering \& Technology, Trivandrum. She is currently working on $\mathrm{Ph} . \mathrm{D}$ in Resource Allocation in Cognitive Radio Networks in A.P.J. Abdul Kalam Technological University.

Her areas of interest includes Wireless Communication, 5G/beyond 5G Systems, Carrier Aggregation, Cognitive Radio, Resource allocation.

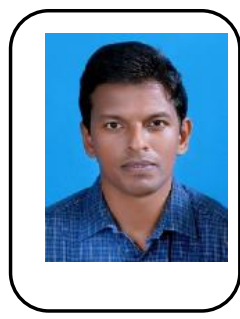

Dr. Sreeni received Ph.D from IIT Bombay in 2013 on the topic of Computational Haptics. In 2003, he obtained M.Tech degree in Power Electronics from NIT, Calicut and B.Tech degree in 2000 from College of Engineering, Adoor. His area of interest includes Linear Algebra for Signal Processing. 